\title{
Formulation and Controlled Release behavior of Urea Fertilizer from biodegradable superabsorbent composite
}

\author{
El-Refaie S. Kenawy ${ }^{1 *}$, Mohamed Rashad ${ }^{2}$, Ali Hosny ${ }^{1}$ \\ 1. Polymer Research Group, Department of Chemistry, Faculty of Science, Tanta University, Tanta, 31527 Egypt \\ 2. Land and Water Technologies Department, Arid Lands Cultivation Research Institute, City of Scientific \\ Research and Technological Applications (SRTA-City), New Borg Elarab, Alexandria, Egypt. \\ * Correspondence: El-Refaie Kenawy, Chemistry Department, Faculty of Science, Tanta University, Tanta \\ 31527, Egypt; ekenawy@yahoo.com.
}

KEY WORDS

Urea, Fertilizers, Controlled release, Biodegradable, superabsorbent composite.

\begin{abstract}
Controlled release technology is one of the most efficient techniques to reduce environmental hazards accompanied with fertilizers and herbicides. In the present work, new formulations for controlled release of urea fertilizers were prepared based on biodegradable polymers and agricultural waste. The release behavior of urea fertilizer from the superabsorbent composite was estimated using UV/Vis spectrophotometer. The effect of the $\mathrm{pH}$ of the soil on the release rate was also investigated.
\end{abstract}

\section{Introduction}

Urea is the most widely used fertilizer globally due to its high nitrogen content (46\%), low cost, and ease of application [1],[2]. Therefore, the development of controlled release coated urea (CRCU) has been a subject of interest for decades [3]. When applied to the soil, urea undergoes a series of biological, chemical and physical transformations to produce the nutrients that is essential to plant [1].

During early growth, plants need only a small quantity of food, so excess nutrients are lost due to leaching. The nitrogen is lost through hazardous gaseous emissions. The production of a suitable CRCU is therefore needed to solve these problems.

Controlled release fertilizer (CRF) is a purposely designed manure that releases active fertilizing nutrients in a controlled, delayed manner in synchrony with the sequential needs of plants for nutrients, thus, they provide enhanced nutrient use efficiency along with enhanced yields [4]. An ideal controlled release fertilizer is coated with a natural or semi-natural, environmentally friendly macromolecule material that retards fertilizer release to such a slow pace that a single application to the soil can meet nutrient requirements for model crop growth [5]. The 
terms, controlled release fertilizer (CRF), and slow release fertilizer (SRF), are generally considered analogous. Nevertheless, Trenkel [1] and Shaviv [4] defined differences between both. In the case of SRFs, the pattern of nutrient release is nearly unpredictable and remains subject to changes in soil type and climatic conditions.

To the contrary, the pattern, quantity, and time of release can be predicted, within limits, for CRFs. A rigorous literature review reveals that the history of CRFs' development and evolution has roots in the early 1960's [6]. Initially, sulfur and polyethylene were used as coating materials in the preparation of SRFs. This history eventually included numerous polymer materials, natural coating agents, multifunctional super-absorbent materials, and even nano-composites. Many of the CRFs have also been prepared on commercial scale so far. In this work, new controlled release urea fertilizer was prepared using biodegradable superabsorbent composite.

\section{Experimental}

\subsection{Materials and Methods}

Superabsorbent composite was prepared previously by Kenawy et al, (submitted to the Arabian journal of chemistry). Sodium hydroxide, hydrochloric acid and urea were purchased from Elnasr pharmaceutical chemicals Co., Egypt.

\subsection{Preparation of the controlled release urea fertilizer}

To prepare the CRUF, a series of samples was prepared according to the following procedure: a weighted quantity of the superabsorbent composite was mixed with a weighted quantity of urea dissolved in distilled water for 24 hours to allow it reaching swelling equilibrium. Thus, all the urea solution is entrapped inside the superabsorbent composite. Afterwards, the swollen composites were dried in a rotary vacuum evaporator at $80{ }^{\circ} \mathrm{C}$ under reduced pressure till water is completely evaporated and all the urea particles are entrapped inside the superabsorbent composite. In turn, CRUF matrix is ready for use. Other CRUF samples were prepared in a similar method with several feed compositions as shown in table (1).

Table (1): Feed composition of the different prepared CRUF samples.

\begin{tabular}{ccc}
\hline Sample code & Urea Wt $\%$ & $\begin{array}{c}\text { Composite } \\
\text { Wt } \%\end{array}$ \\
\hline CRUF1 & 90 & 10 \\
CRUF2 & 80 & 20 \\
CRUF3 & 70 & 30 \\
CRUF4 & 60 & 40 \\
CRUF5 & 50 & 50 \\
\hline
\end{tabular}

\subsection{Study of urea release from the CRUF matrix in water}

The release behavior of urea from the CRUF in distilled water was studied according to the following procedure: $0.3 \mathrm{~g}$ of the CRUF was contained in a tea bag-like tissue and then immersed in $200 \mathrm{ml}$ of distilled water in a glass beaker properly covered and kept at $25{ }^{\circ} \mathrm{C}$. After one hour, $10 \mathrm{ml}$ of water in the glass beaker were withdrawn to measure the amount of urea released from the matrix and the same volume of fresh water was replenished. Detection of released urea was performed using UV-Vis spectrophotometer. According to Ehrlich reaction, $\mathrm{P}-\mathrm{N}, \mathrm{N}^{\prime}$ dimethylamino benzaldehyde reacts with urea to give a lemon Kelly color which would absorb visible light at $440 \mathrm{~nm}$. All the release experiments were done in a triplicate, and their results were averaged.

\subsection{Release of urea from CRUF in soil}

In order to demonstrate the release of urea from superabsorbent composite in agricultural field, release experiments in soil column were done. The soil used in this experiment was airdried and sieved to less than $2 \mathrm{~mm}$. Polyvinyl chloride (PVC) pipe of $45 \mathrm{~mm}$ internal diameter was used to hold soil. Loaded CRUF ( 2 g) was well mixed with 200 g dry sandy soil and placed in the PVC pipe. The bottom of the tube was sealed by nylon fabric. Water was added slowly by a perfusion tube (one drop every $10 \mathrm{~s}$ ). The time started when water seeped from the bottom of the pipe, i.e. water saturation. The leached water was collected, from which urea was estimated by the ultraviolet spectrophotometer [7]. Control experiments, i.e. urea, (the amounts of urea 
were the same as that of loaded CRUF) were also carried out, respectively.

\section{Results and discussions}

\subsection{Release behavior of urea from the controlled release fertilizer (CRUF)}

The study of the release of urea from superabsorbent composite was carried out as described in the experimental part. The best composite-urea formulation that can give good controlled release behavior was selected. To investigate the properties of the selected composite as a new controlled release system, various formulations were prepared from the selected composite and urea as shown in table (1). The release measurements were carried out in distilled water at room temperature $\left(25{ }^{\circ} \mathrm{C}\right)$ and the release was monitored using UV spectrophotometer at $440 \mathrm{~nm}$.
The studies were carried out for 16 hours the results showed clearly that on increasing the amount of composite in the formulation from $10 \mathrm{wt} \%$ to $50 \mathrm{wt} \%$, the release rate decrease and release time increased from 3 hours (in case of $\mathrm{CRUF}_{1}$ ) to 16 hours (in case of $\mathrm{CRUF}_{5}$ ).

Figure (1) depicts the release profiles of urea from loaded controlled release urea fertilizer (CRUF) with different urea loading percentage in distilled water (swollen in aqueous solutions of urea with different concentrations). Even though pure urea dissolve in water entirely in five seconds, $\mathrm{CRUF}_{1}$ released $100 \%$ of its urea after 3 hours while the $\mathrm{CRUF}_{2}$ released $100 \%$ of urea after 6 hours. Similar results were obtained for $\mathrm{CRUF}_{3}, \mathrm{CRUF}_{4}$ and $\mathrm{CRUF}_{5}$ which released $100 \%$ of urea in 10,13 and 16 hours, respectively.

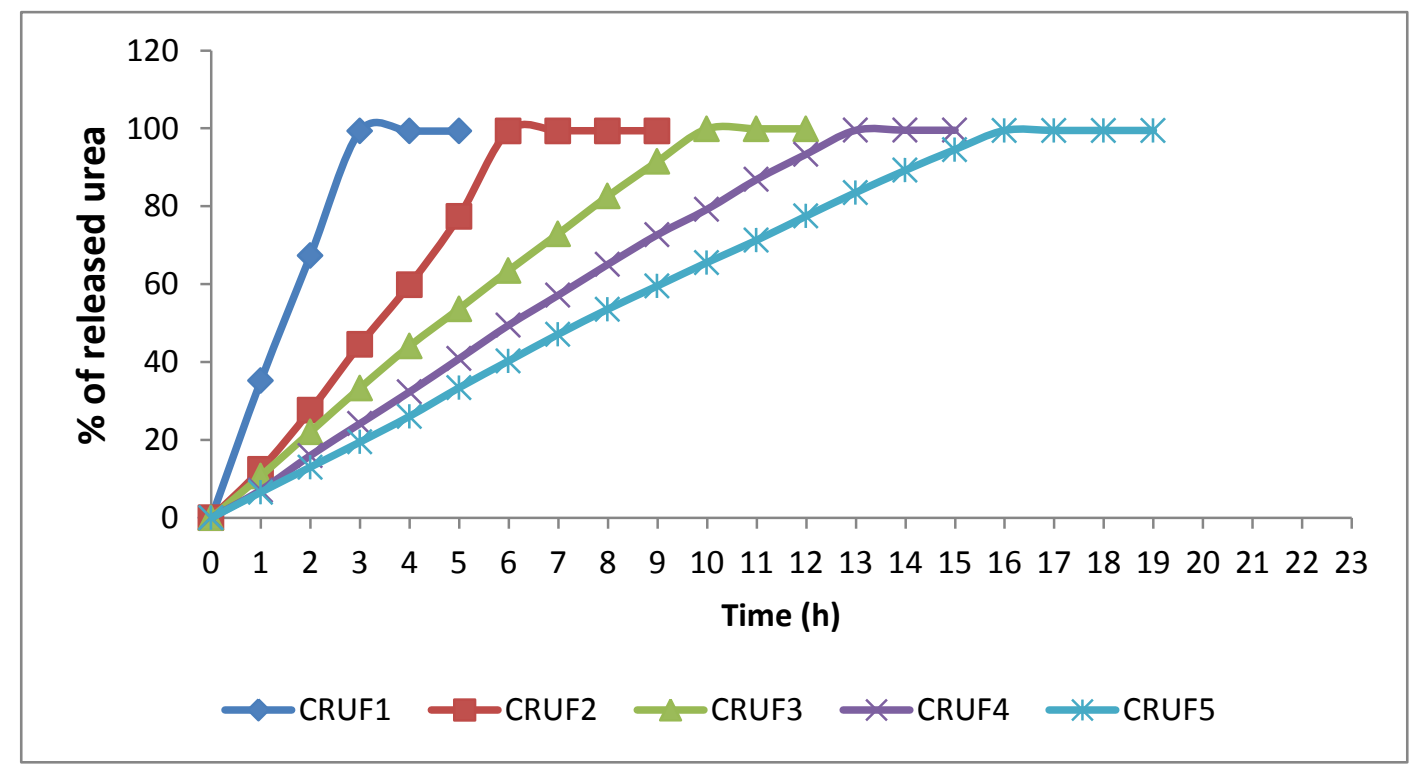

Figure (1): The release rate of urea from different CRUF formulations in distilled water.

It was found that during the first hour of release study, the CRUF1 released $35 \%$ of urea, while $\mathrm{CRUF}_{2}$ released $12.5 \%$ of its urea content. The decrease of percentage of the released urea was repeated with the other formulations. The first hour release of $\mathrm{CRUF}_{3}$, $\mathrm{CRUF}_{4}$ and $\mathrm{CRUF}_{5}$ were $10.8 \%, 7 \%$ and $6.5 \%$ respectively. The high percent of the released urea from CRUF $_{1}$ could be due to the release of urea adsorbed on the composite surface and pores formed on the surface CRUF.
The formed pores on the surface may have led to easy penetration of water vapor to the urea entrapped in the core causing the initial release of urea in the first hour of release study as the coating composite has not reached the swelling equilibrium yet. After the swelling equilibrium is reached, all the pores on the surface may have been closed forcing urea release from the CRUF to follow a diffusion mechanism from the interlayers of the composite. 
The decrease in the rate of the released ureacomposite formulation was noted by increasing the superabsorbent composite content. This could be attributed to the fact that the release is diffusion controlled. Therefore, on increasing the percent of superabsorbent composite, more barriers are formed which decreases the diffusion rate and consequently the release rate of urea. After the swelling equilibrium was achieved, the perfect swollen samples were formed.

\subsection{The effect of $\mathrm{pH}$ on the release of urea from the controlled release fertilizer (CRUF5)}

To investigate the effect of the $\mathrm{pH}$ of the release media (aqueous media) on the release rate of urea, the release was conducted in the different $\mathrm{pHs}$ media ( $\mathrm{pH} 4,7$ and 10).

Figure (2) shows that there was a slight difference in the release behavior of urea from the $\mathrm{CRUF}_{5}$ in the various $\mathrm{pHs}$ that $100 \%$ released after 11,16 and $14 \mathrm{~h}$ for $\mathrm{pHs} 4,10$ and 7 respectively. This could be attributed to the presence of different ions. The presence of cations like $\mathrm{Na}^{+}, \mathrm{Ca}^{+2}, \mathrm{Mg}^{+2}, \mathrm{Fe}^{+3}, \mathrm{H}^{+}$and others lowered the swelling of the composite involved in CRUF5 formulation due to the screening effect of these cations on the anionic hydrophilic groups, which restricts the expanding of polymeric network and consequently increase the release rate of urea from the urea-composite formulation.

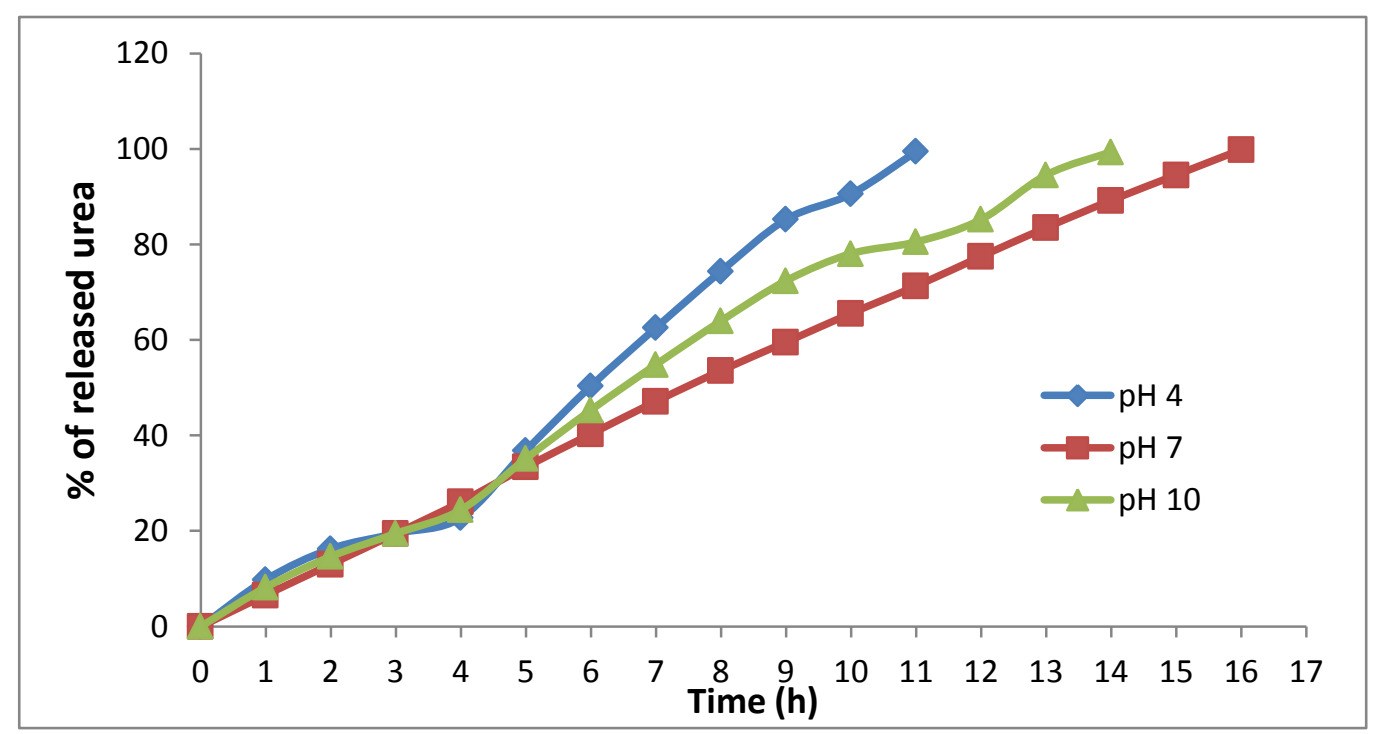

Figure (2): The release rate of urea from CRUF5 in different media.

\subsection{Release of urea from CRUF in soil}

Because there are many factors that could affect the release of urea from loaded CRUF in soil, such as the kind of soil, cation exchange capacity (CEC), water content, bulk density, urea concentration, gel collapse and so on, there must exist obvious differences for urea release from loaded CRUF between in distilled water and in soil. Therefore, we measured the urea release behaviors in soil.

Figure (3) shows the urea release behaviors of loaded CRUF and untreated urea in soil. The urea release rate of untreated urea was very fast (as shown in figure (3). While the urea release rate of loaded CRUF decreased obviously compared with that of urea, the result was in agreement with that of Smith [8], as shown also in Figure (3), the urea released $44.5 \%, 68.4 \%$ and $99.5 \%$ within 1,5 and 10 days, respectively. The urea release characters of loaded CRUF in soil was similar to that in water except that the release rate was slower in soil. It is well known that urea is easily dissolved in water, so it would quickly dissolve in the soil solution, and then release out of the soil by purge water, therefore, the release rate of untreated urea was very fast. However, for the loaded CRUF, the release of urea could be described by the following steps:

1)CRUF was slowly swollen by the water in soil and then transformed to hydrogel, and urea dissolved. 
2) Urea could be released or desorbed slowly through the dynamic exchange of free water between soil solution and CRUF [9].

3) The released urea was purged out of the soil by water.

The reasons for the slower release rate in soil than in water may be due to the existing of many kinds of ions in soil solution, the swelling degree of CRUF is less in soil than that in distilled water, then the diffusion of urea in it would be difficult [10]. Moreover, when the urea passed through the soil column, there are many-time absorbing and deabsorbing processes between urea molecule and soil granules, which also contribute to the slower urea release rate in soil.

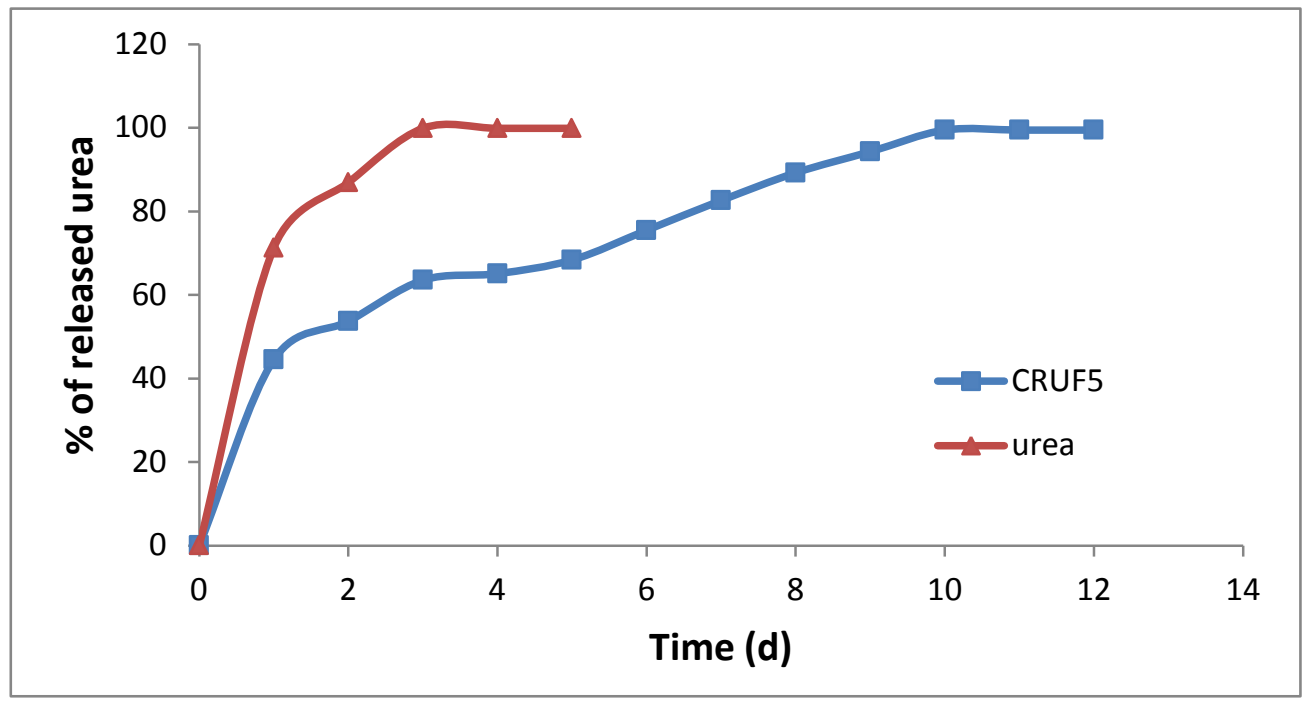

Figure (3): The release rate of urea from CRUF5 in the soil.

\section{Conclusion}

The release study of urea from the formulated controlled release urea fertilizer (CRUF) showed that the rate of urea release from the formulated CRUF decreased with the increased composite content while $\mathrm{pH}$ had no evident effect on the release rate. The prepared CRUF showed that urea could last in soil for nearly 12 days while pure urea disappeared from soil after about 3 days only.

\section{Acknowledgment}

The authors extend their appreciation to the Science and Technology Development Fund (STDF) for funding this work through research project entitled "Superabsorbent polymer composite for agricultural applications" (Project ID: 5842).

\section{References}

[1] M. Trenkel, Slow- and Controlled-Release and Stabilized Fertilizers: An Option for Enhancing Nutrient Use Efficiency in Agriculture, Second edi., vol. 1. Paris, France: International Fertilizer Industry Association (IFA), 2010.

[2] T. Zheng, Y. Liang, S. Ye, and Z. He, "Superabsorbent hydrogels as carriers for the controlled-release of urea: Experiments and a mathematical model describing the release rate," Biosyst. Eng., vol. 102, no. 1, pp. 44-50, 2009.
[3] D. W. Rindt, G. M. Blouin, and J. G. Getsinger, "Sulfur coating on nitrogen fertilizer to reduce dissolution rate," J. Agric. Food Chem., vol. 16, no. 5, pp. 773-778, 1968.

[4] A. Shaviv, "Controlled release fertilizers," in IFA International Workshop on Enhanced-Efficiency Fertilizers, 2005.

[5] G. M. Blouin and D. W. Rindt, "Method of Making Sulfur-Coated Fertilizer Pellet Having a Controlled Dissolution Rate, " 1967.

[6] O. E. M. Glenn M. Blouin, Donald W. Rindt, "Sulfur-coated fertilizers for controlled release. Pilotplant production," Agric. Food Chem., vol. 19, no. 5, pp. 801-808, 1971.

[7] G. W. Watt and J. D. Chrisp, "Spectrophotometric method for determination of urea," Anal. Chem., vol. 26, no. 3, pp. 452-453, 1954.

[8] J. D. Smith and H. C. Harrison, "Evaluation of polymers for controlled release properties when incorporated with nitrogen fertilizer solutions," Commun. Soil Sci. Plant Anal., vol. 22, no. 5-6, pp. 559-573, 1991.

[9] D. S. G. Hu and M. T. S. Lin, "Water-polymer interactions and critical phenomena of swelling in inhomogeneous poly(acrylonitrile-acrylamide-acrylic acid) gels," Polymer (Guildf)., vol. 35, no. 20, pp. 4416-4422, 1994.

[10] A. K. Bajpai and A. Giri, "Swelling dynamics of a macromolecular hydrophilic network and evaluation of its potential for controlled release of agrochemicals," React. Funct. Polym., vol. 53, no. 2-3, pp. 125-141, 2002. 